\title{
Ictal-interictal continuum: a review of recent advancements
}

\author{
James X. Tao ${ }^{1 *}$, Xiaoxiao Qin ${ }^{2,3}$ and Qun Wang ${ }^{2,3}$
}

\begin{abstract}
Continuous electroencephalogram (cEEG) has become an indispensable technique in the management of critically ill patients for early detection and treatment of non-convulsive seizures (NCS) and non-convulsive status epilepticus (NCSE). It has also brought about a renaissance in a wide range of rhythmic and periodic patterns with heterogeneous frequency and morphology. These patterns share the rhythmic and sharp appearances of electrographic seizures, but often lack the necessary frequency, spatiotemporal evolution and clinical accompaniments to meet the definitive criteria for ictal patterns. They may be associated with cerebral metabolic crisis and neuronal injury, therefore not clearly interictal either, but lie along an intervening spectrum referred to as ictal-interictal continuum (IIC). Generally speaking, rhythmic and periodic patterns are categorized as interictal patterns when occurring at a rate of $<1 \mathrm{~Hz}$, and are categorized as NCS and NCSE when occurring at a rate of $>2.5 \mathrm{~Hz}$ with spatiotemporal evolution. As such, IIC commonly includes the rhythmic and periodic patterns occurring at a rate of 1-2.5 Hz without spatiotemporal evolution and clinical correlates. Currently there are no evidence-based guidelines on when and if to treat patients with IIC patterns, and particularly how aggressively to treat, presenting a challenging electrophysiological and clinical conundrum. In practice, a diagnostic trial with preferably a non-sedative anti-seizure medication (ASM) can be considered with the end point being both clinical and electrographic improvement. When available and necessary, correlation of IIC with biomarkers of neuronal injury, such as neuronal specific enolase (NSE), neuroimaging, depth electrode recording, cerebral microdialysis and oxygen measurement, can be assessed for the consideration of ASM treatment. Here we review the recent advancements in their clinical significance, risk stratification and treatment algorithm.
\end{abstract}

Keywords: Periodic discharges, Critical care, Continuous EEG, Ictal-interictal continuum, Nonconvulsive seizures

\section{Background}

Periodic discharges were initially described by Cobb and Hill in patients with subacute progressive encephalitis in 1950 [1]. Chatrian and colleagues later described periodic lateralized epileptiform discharges (PLEDs) in patients with acute focal brain lesions in 1964 [2]. In the light of widespread use of continuous EEG monitoring for critically ill patients in the last several decades, a spectrum of rhythmic and periodic patterns have been described. The American Clinical Neurophysiology Society (ACNS) has created a uniform EEG terminology for

\footnotetext{
* Correspondence: jtao@neurology.bsd.uchicago.edu

${ }^{1}$ Department of Neurology, The University of Chicago, 5841 South Maryland Ave. MC2030, Chicago, IL 6063, U.S.A.

Full list of author information is available at the end of the article
}

describing these EEG patterns in critically ill patients, aiming to classify these patterns with a universal nomenclature, improve interrater reliability and facilitate research $[3,4]$. Under the ACNS standardized critical care EEG terminology, rhythmic and periodic patterns are classified into three different subcategories: 1) periodic discharges (PDs), 2) rhythmic delta activity (RDA), and 3) spike or sharp wave discharges (SW). These patterns can be further classified as generalized, lateralized, bilateral independent and multifocal, such as generalized periodic discharges (GPDs), generalized rhythmic delta activity (GRDA), lateralized periodic discharges (LPDs), lateralized rhythmic delta activity (LRDA), and bilateral independent periodic discharges (BIPDs) [5].

(c) The Author(s). 2020 Open Access This article is licensed under a Creative Commons Attribution 4.0 International License, which permits use, sharing, adaptation, distribution and reproduction in any medium or format, as long as you give appropriate credit to the original author(s) and the source, provide a link to the Creative Commons licence, and indicate if changes were made. The images or other third party material in this article are included in the article's Creative Commons licence, unless indicated otherwise in a credit line to the material. If material is not included in the article's Creative Commons licence and your intended use is not permitted by statutory regulation or exceeds the permitted use, you will need to obtain permission directly from the copyright holder. To view a copy of this licence, visit http://creativecommons.org/licenses/by/4.0/. 
The concept of IIC was first coined by PohlmannEden et al. in 1996, who described PLEDs as "an electrographic signature of a dynamic pathophysiological state in which unstable neurobiological processes create an ictal interictal continuum, with the nature of the underlying neuronal injury, the patient's preexisting propensity to have seizures, and the coexistence of any acute metabolic derangements all contributing to whether seizures occur or not" [6]. The current use of ictal-interictal continuum has been expanded to include other rhythmic and periodic patterns (i.e. LPDs, GPDs, BIPDs, LRDA and GRDA) [7, 8]. Nevertheless, there is no consensus agreement on the definition of IIC. IIC commonly includes the rhythmic and periodic patterns occurring at a rate of $1-2.5 \mathrm{~Hz}$ without spatiotemporal evolution and clinical correlates [9]. Brief potentially ictal rhythmic discharges are $>4 \mathrm{~Hz}$ and $<10 \mathrm{~s}$. They do not meet the criteria for an ictal pattern [10], and thus can be considered IIC patterns.

\section{EEG patterns of ictal-interictal continuum} Lateralized periodic discharges

LPDs are formerly referred to as PLEDs. They are the most commonly observed periodic pattern, seen in 4.7 to $8.6 \%$ of critically ill patients $[9,11-14]$. These discharges have a sharp or spiky morphology, and are typically $100-300 \mu \mathrm{V}$ in amplitude (Fig. 1) [4]. LPDs are commonly seen in patients with structural brain injuries, including acute stroke, traumatic brain injury, encephalitis, and tumors [11, 12, 15-18]. Acute stroke is by far the most common etiology [11, 19]. Data from rats with focal ischemia suggested that LPDs were originated specifically from the ischemic penumbra rather than the infarcted core tissue [20]. LPDs may also occur in the setting of epilepsy, systemic infection, metabolic, and toxic insults as well as in the absence of structural lesions [15]. LPDs are significantly associated with an increased risk of seizures in $40-90 \%$ of patients during cEEG monitoring [11, 13, 16, 21]. When associated with plus features, LPDs were more likely to be correlated with seizures [12, 22]. A greater risk of seizures was also associated with LPDs $>2 \mathrm{~Hz}$ [22]. They are also independently associated with increased rates of severe disability, vegetative state, and death, with mortality rates ranging from 25 to $41 \%$ [12, 13, 17, 19, 23, 24].

\section{Generalized periodic discharges}

GPDs are generalized waveforms that have relatively uniform morphology and duration with a quantifiable inter-discharge interval between consecutive waveforms at nearly regular intervals (Fig. 2) [4, 25]. Historically, GPDs were referred to as GPEDs. Triphasic waves (TW) are included in the GPDs, and often described as GPDs with triphasic morphology $[4,26]$. The prevalence of GPDs varied from 0.8 to $4.5 \%$ in critically ill patients. Common etiologies include toxic-metabolic encephalopathy, anoxic brain injury, acute brain injury, infections and epilepsy [25, 27]. GPDs are often associated with

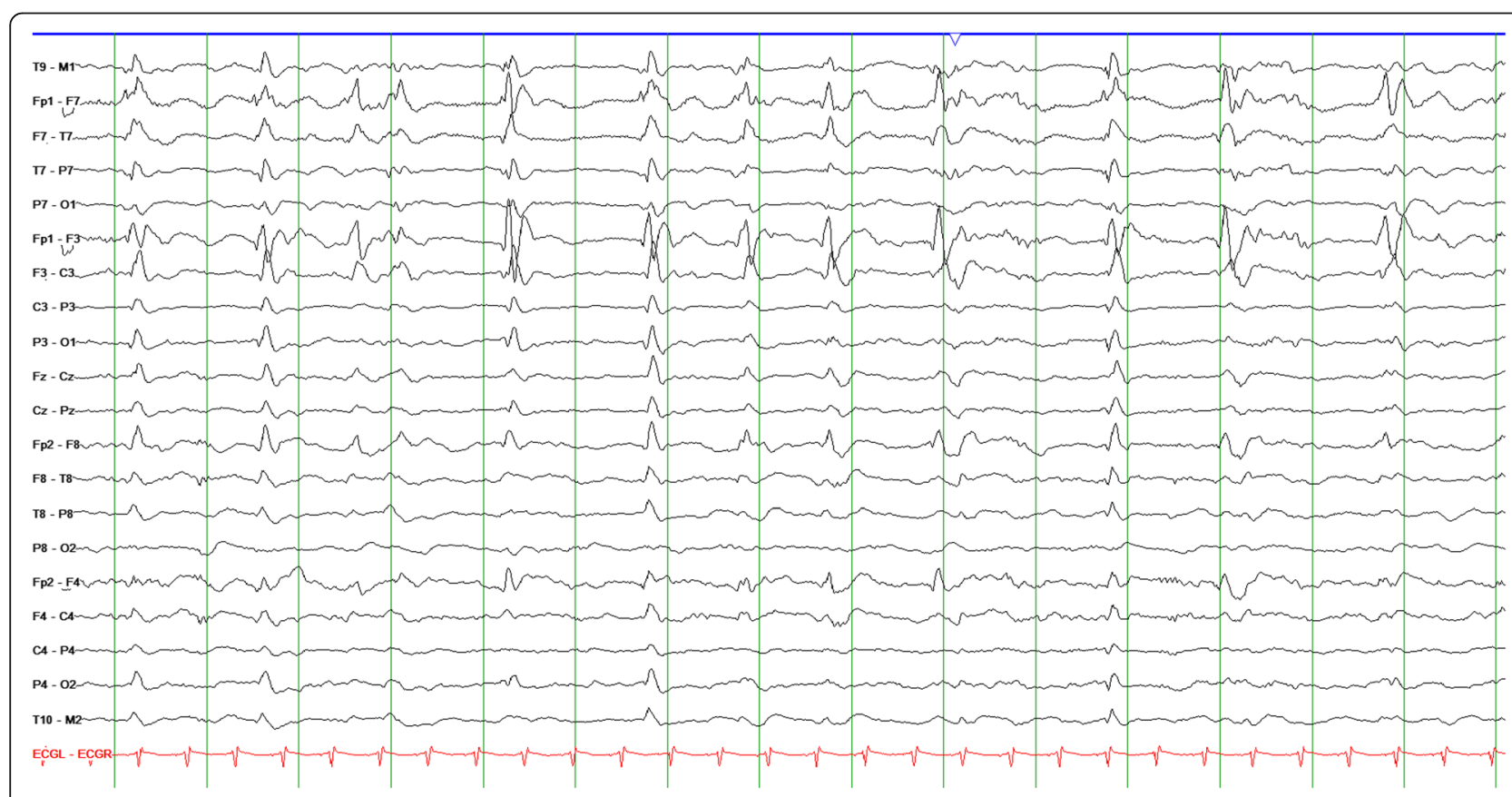

Fig. 1 Lateralized periodic discharges (LPDs) at a frequency of approximately $1 \mathrm{~Hz}$ in a patient with hemorrhagic stroke in the left fronto-temporal region. High pass filter $1 \mathrm{~Hz}$, low pass filter $50 \mathrm{~Hz}$ and sensitivity $10 \mathrm{uV} / \mathrm{mm}$ 


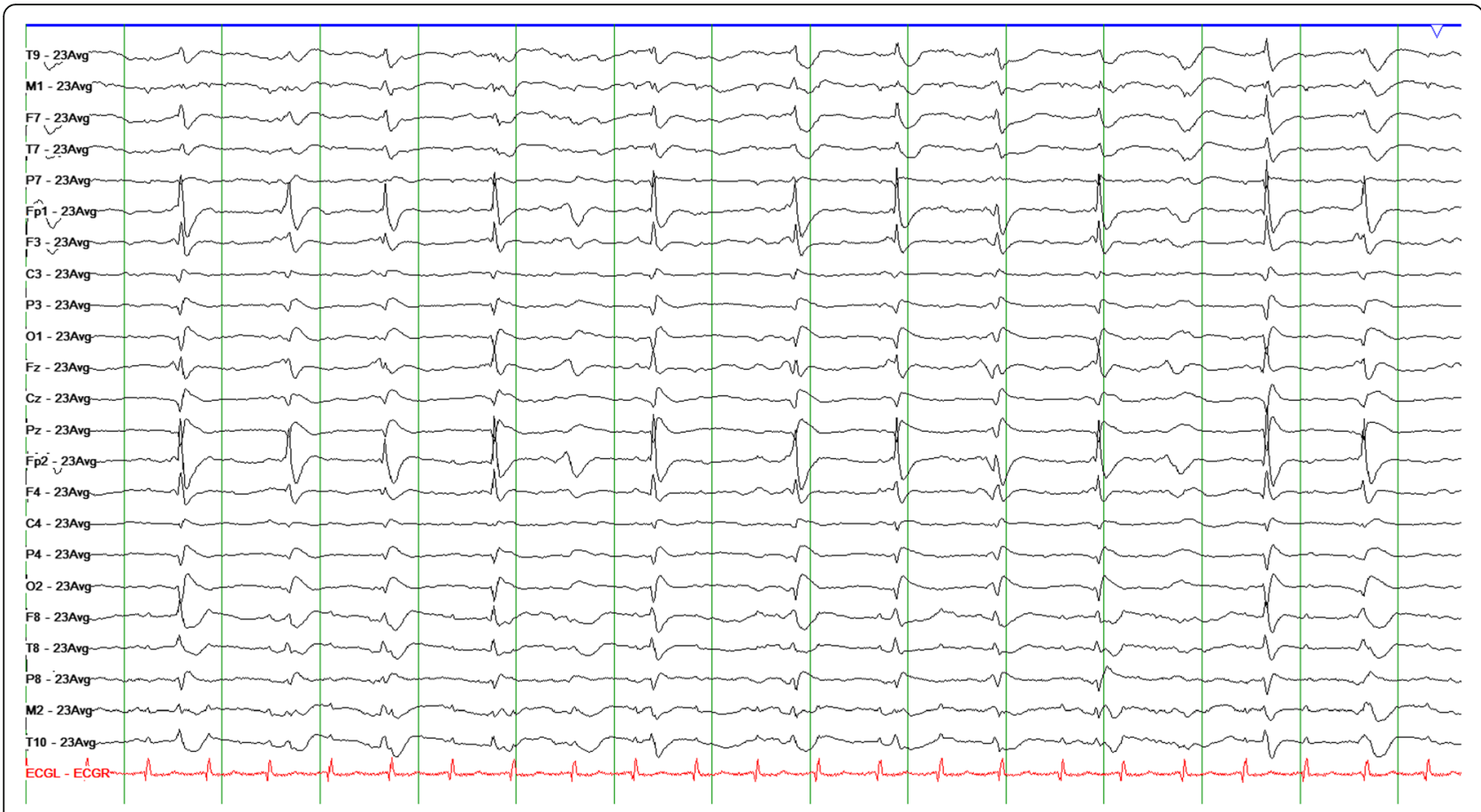

Fig. 2 Generalized periodic discharges (GPDs) at a frequency of approximately $1 \mathrm{~Hz}$ in a patient with anoxic brain injury. High pass filter $1 \mathrm{~Hz}$, low pass filter $50 \mathrm{~Hz}$ and sensitivity $10 \mathrm{uV} / \mathrm{mm}$

seizures, particularly NCS and NCSE. In a retrospective case-control study of 200 patients, $27 \%$ of patients with GPDs had NCS, and $22 \%$ had NCSE, compared with $8 \%$ with NCS and 7\% with NCSE among 200 matched controls [25]. In a more recent retrospective study of 4772 patients undergoing cEEG monitoring, high-frequency $(>1.5 \mathrm{~Hz}$ ) GPDs were more likely to be associated with seizures [22]. Whether GPDs predict worse clinical outcomes remains controversial. In one study, only $36 \%$ of patients with GPDs were alive at the time of discharge [28]. However, a more recent study showed that after controlling for age, etiology, and the level of consciousness, GPDs were not an independent marker for poor prognosis [25].

\section{Bilateral independent periodic discharges}

BIPDs are the lateralized periodic discharges that occur independently over each hemisphere [4]. BIPDs are previously known as bilateral independent periodic lateralized epileptiform discharges (BI-PLEDs). The prevalence of BIPDs is much less frequent than LPDs, approximately $0.5-1 \%$ in critically ill patients undergoing CEEG monitoring [29]. They typically occur with acute bilateral or diffuse cerebral injury such as traumatic brain injury, stroke, infections and anoxic encephalopathy $[2,12,13,30]$. The high risk of electrographic seizures associated with BIPDs has been reported in several studies [2, 12, 13, 29, 31].

\section{Lateralized rhythmic delta activity}

LRDA is characterized by rhythmic delta activity predominantly involving one hemisphere (Fig. 3). The prevalence of this pattern is seen in 4.7 to $7.1 \%$ of critically ill subjects underdoing continuous EEG [21, 22, 32]. The most common causes of LRDA in critically ill patients were intracerebral hemorrhage and subarachnoid hemorrhage. LRDA has a similar clinical significance as lateralized periodic discharges in critically ill patients, and is associated with a high risk of acute seizures, especially nonconvulsive seizures [21]. It was found that seizures developed in $28-63 \%$ patients with LRDA, and seizures were significantly more likely with LRDA greater than $2 \mathrm{~Hz}$ or with a "plus" modifier (i. e. LRDA + R or LRDA +S) [21, 22].

LRDA is also commonly found in patients with temporal lobe epilepsy without focal structural abnormalities and is formerly referred to as temporal intermittent rhythmic delta activity (TIRDA) [33, 34]. LRDA is strongly associated with the presence of temporal lobe spikes or sharp waves [35]. LRDA has also been proposed as a marker for mesial temporal atrophy. Seventy-five percent of patients with TIRDA have 


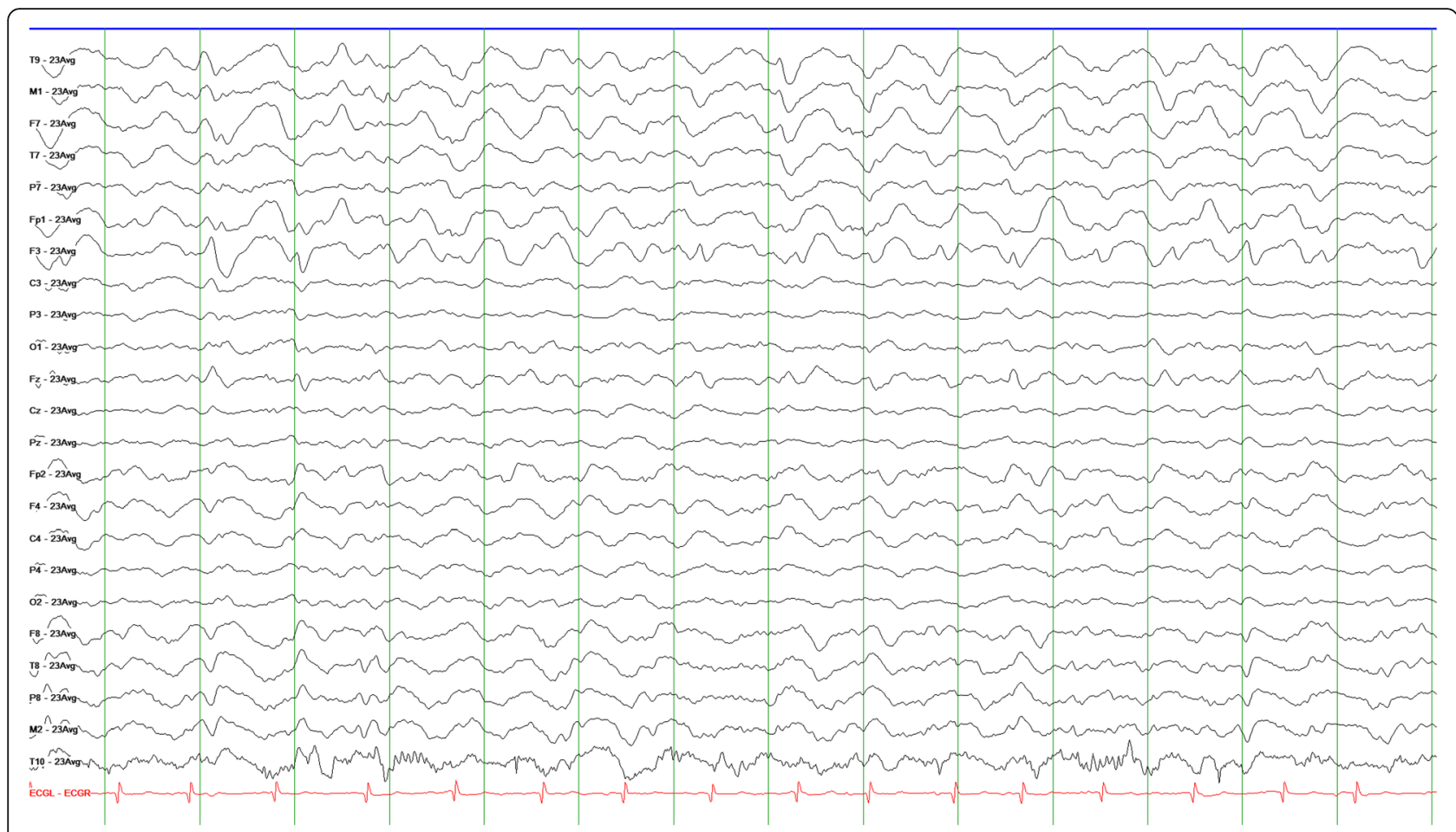

Fig. 3 Lateralized rhythmic delta activity (LRDA) at a frequency of $0.5-1 \mathrm{~Hz}$ in a patient with metabolic derangement. High pass filter $1 \mathrm{~Hz}$, low pass filter $50 \mathrm{~Hz}$ and sensitivity $10 \mathrm{uV} / \mathrm{mm}$

evidence of signal abnormalities in the mesial temporal structures and $86 \%$ of cases of pathologically determined mesial temporal sclerosis show TIRDA on EEG [35].

\section{Generalized rhythmic delta activity}

GRDA is defined as $1-4 \mathrm{~Hz}$ generalized delta activity that is relatively uniform in morphology and duration without interval between consecutive waveforms (Fig. 4) [4]. GRDA is formerly referred to as frontal intermittent rhythmic delta activity (FIRDA) or occipital intermittent rhythmic delta activity (OIRDA). GRDA has been reported in a wide variety of cerebral lesions and metabolic disturbances [32]. Unlike most other rhythmic and periodic patterns, GRDA does not appear to be associated with seizures $[22,36,37]$. However, in a study of 665 critically ill patients, electrographic seizures were observed in 4 of 40 (10\%) patients with GRDA [29]. GRDA is commonly not associated with poor clinical outcomes at the time of hospital discharge [38-40].

\section{Stimulus-induced rhythmic, periodic or ictal discharges}

Stimulus-induced rhythmic, periodic or ictal discharges (SIRPIDs) are hyperexcitable discharges commonly seen during continuous EEG recordings of critically ill patients, and are elicited by stimulation (e.g., suctioning, turning, bed-side nursing care) [41]. SIRPIDs can be lateralized or generalized periodic patterns. They are present in 10 to
$34 \%$ of hospitalized patients being monitored on cEEG [41-43]. Common causes of SIRPIDs include anoxic brain injury, metabolic encephalopathy, subarachnoid hemorrhage, CNS infections, and creutzfeld-Jacob disease [44]. The clinical implication of SIRPIDs remains uncertain. Some studies suggest a strong association between SIRPIDs and seizures in critically ill patients, and particularly in those with acute brain injury $[4,41]$. However, in a large, multicenter study of 4772 patients undergoing cEEG monitoring, LPDs, LRDA, and GPDs were significantly associated with seizures, but whether the underlying pattern was stimulus-induced or not had no effect on the association with seizures [22].

\section{Brief potentially ictal rhythmic discharges}

Brief potentially ictal rhythmic discharges or B(I) RDs are defined as lateralized rhythmic discharges of $>4 \mathrm{~Hz}$ in theta, alpha and beta frequency and lasting $<10 \mathrm{~s}$ with or without evolution [10]. The prevalence of $\mathrm{B}(\mathrm{I}) \mathrm{RDs}$ ranges from 17 to $20 \%$ in neonates [45-47], and in $2 \%$ critically ill patients who received urgent cEEG [10]. This pattern is commonly seen patients with acute brain injury and anti-NMDA encephalitis, and is associated with an increased risk of acute seizures in up to $75 \%$ critically ill patients $[10,48]$. B(I) RDs were also found in 15 of 1230 patients (1.2\%) with epilepsy undergoing long-term EEG monitoring, particularly in patients with 


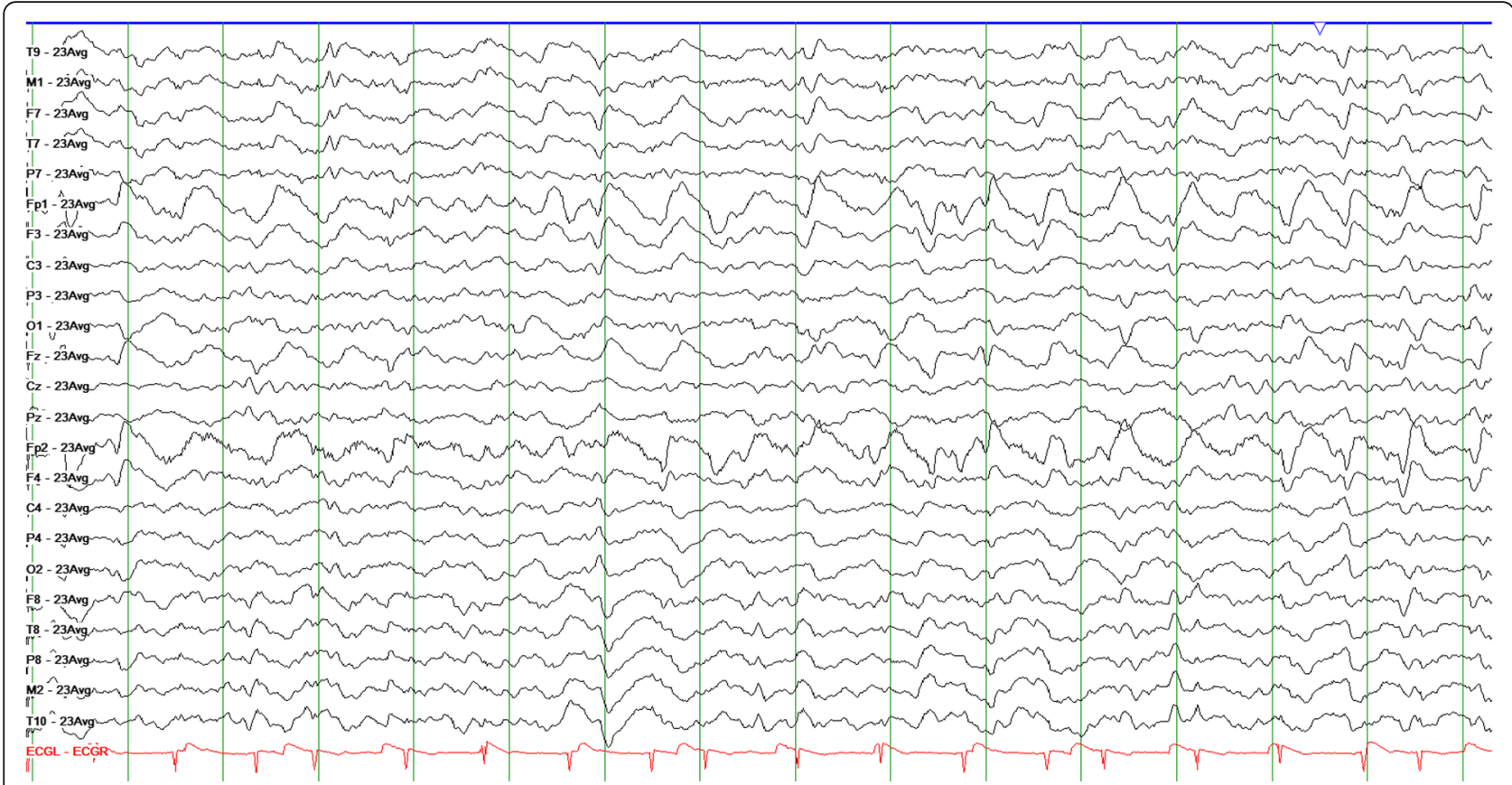

Fig. 4 Generalized rhythmic delta activity (GRDA) at a fregency of 0.5-1 Hz in a patient with metabolic derangement. High pass filter $1 \mathrm{~Hz}$, low pas filter $50 \mathrm{~Hz}$ and sensitivity $10 \mathrm{uV} / \mathrm{mm}$

mediation resistant epilepsy [49]. B(I) RDs were collateralized with focal cerebral lesion and morphologically similar to EEG seizure onset pattern. Patients with $\mathrm{B}(\mathrm{I})$ RDs tended to have a worse outcome than controls, however, this finding was not statistically significant [10].

\section{Risk stratification of IIC patterns associated with seizures}

The seizure risk associated with IIC patterns can be stratified in the several different perspectives including: (1) segregate IIC patterns from NCS and NCSE; (2) assess the risk of seizures associated with IIC patterns; (3) determine the ictal nature of IIC patterns; (4) assess the long term risk of developing chronic epilepsy.

\section{Differentiate IIC patterns from electrographic seizures}

The first step is to differentiate IIC patterns from NCS or NCSE. Salzburg criteria have been widely adopted to identify NCS or NCSE based on EEG patterns. In order to be considered NCS or NCSE, at least one of the following criteria must be met and be continuously present for at least $10 \mathrm{~s}$ for NCS and $30 \mathrm{~min}$ for NCSE: (1) epileptiform patterns occurring at $>2.5 \mathrm{~Hz}$; (2) concurrent subtle clinical accompaniments; or (3) spatiotemporal evolution [50]. Periodic and rhythmic patterns occurring at a frequency of $<1 \mathrm{~Hz}$ without plus features and displaying fluctuation are generally considered interictal patterns. Patterns occurring at a frequency of $1-2.5 \mathrm{~Hz}$ are considered ictal-interictal continuum. ACNS defines electrographic seizures as "repetitive generalized or focal spikes, sharp waves, spike and wave or sharp-and-slow wave complexes at $\geq 3 \mathrm{~Hz}$, or sequential rhythmic, periodic, or quasi-periodic waves at $\geq 1 \mathrm{~Hz}$ and unequivocal evolution (gradual increase or decrease $\geq 1 \mathrm{~Hz}$ ) [4, 7]. Both Salzburg and ACNS criteria have been used to define the electrographic seizures in clinical practice.

\section{Risk of electrographic seizures associated with IIC}

The increased risk of seizures associated with IIC patterns has been well-described. Morphology, duration and frequency of IIC patterns are highly predictive of the seizure risk [51]. Overall, the incidence of seizures in critically ill patients range from 45 to $95 \%$ in patients with LPDs (Fig. 5) [11-13, 21, 29], 43-78\% in patients with BIPDs [13, 31], 11-89\% in patients with GPDs [11, $25,28,29,52]$, and $35-63 \%$ in patients with LRDA [21, 29]. GRDA was commonly not associated with an increased seizure risk [22]. However, in a study of 665 critically ill patients, electrographic seizures were observed in 4 of $40(10 \%)$ patients with GRDA [29]. Additionally, IIC patterns with plus features were more likely to be associated with seizures. "LPD+ R" was associated with the highest odds for developing seizures and status epilepticus. "LPD+ F" were also highly associated with ictal activity, whereas LPDs bearing blunt morphology were not associated with ictal activity [16]. In generally, GRDA lies at the interictal end of IIC spectrum, whereas LPDs lay at the ictal end of IIC spectrum. Patterns including GPDs, LRDA and $\mathrm{B}(\mathrm{I})$ RDs are in the middle range of 


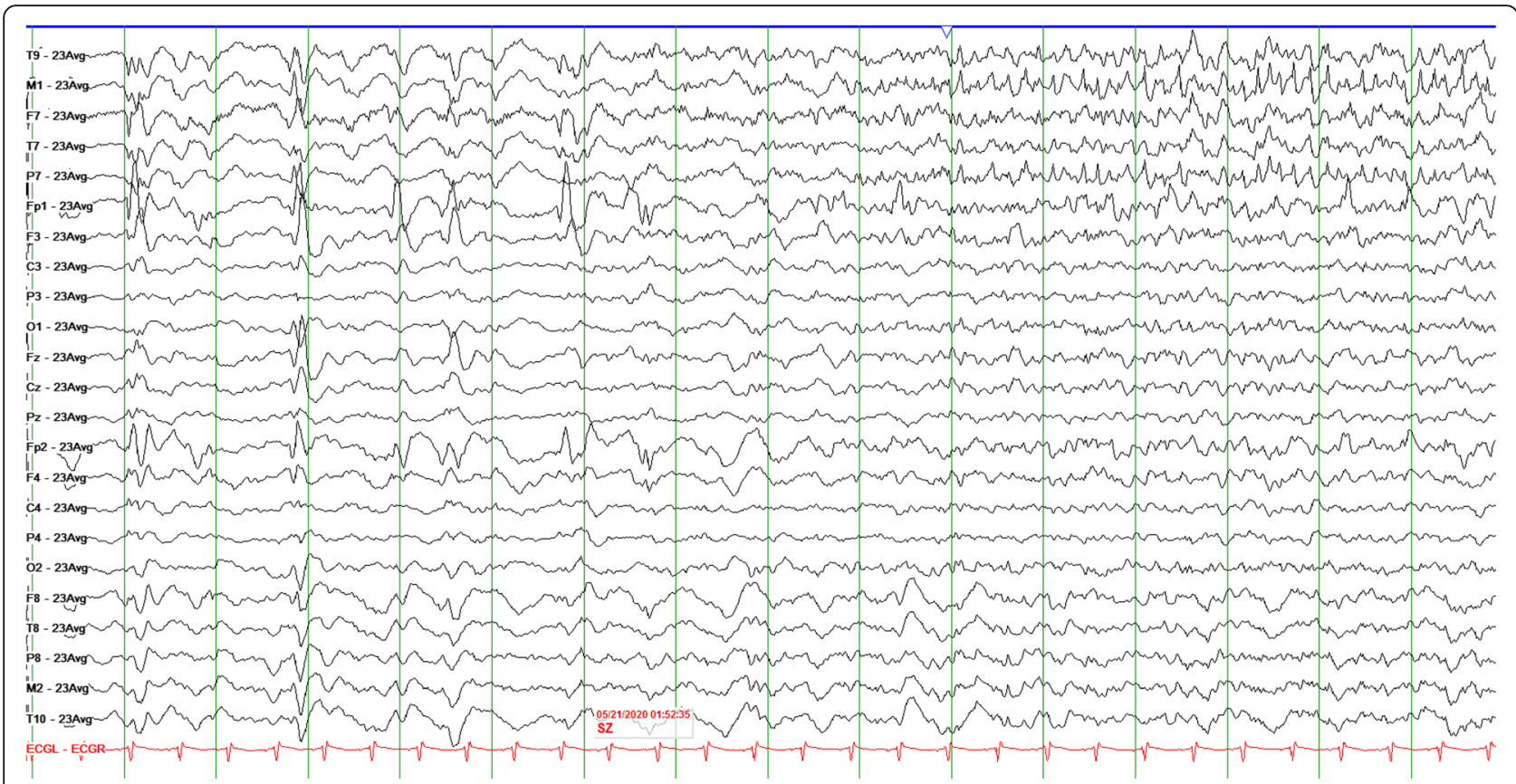

Fig. 5 Lateralized periodic discharges (LPDs) evolving into a seizure in the left frontotemporal region from the same patient as in Figure 1. High pass filter $1 \mathrm{~Hz}$, low pass filter $50 \mathrm{~Hz}$ and sensitivity $10 \mathrm{uV} / \mathrm{mm}$

IIC spectrum for the seizure risk [51, 53]. IIC patterns with frequencies $>1.5 \mathrm{~Hz}$ were associated with an increased seizure risk [22].

\section{IIC patterns being potentially ictal patterns}

It is highly controversial whether IIC patterns are potentially ictal patterns and if they cause neuronal injury. There are several lines of evidence suggesting that IIC patterns might be ictal in nature.

1) LPDs with negative clinical correlates are potentially ictal [54]. When LPDs are correlated with time-locked motor symptoms; LPDs are typically localized to lesions in the motor cortex and are considered ictal. However, when LPDs are correlated with negative clinical symptoms such as aphasia, amnesia, apraxia, and cortical blindness, LPDs are localized to brain regions that do not produce positive clinical symptoms, and are often considered non-ictal. Sen-Gupta and colleagues contended that the difference between ictal and non-ictal LPDs appears to reflect the difference in underlying anatomical locations of the periodic discharges rather than in providing distinction between ictal and non-ictal states [54].

2) IIC patterns may be associated with known imaging markers of electrographic seizures. In several small case studies, IIC patterns were found to be associated with restricted diffusion on diffusion weighted imaging (DWI) on MRI [55], increased regional cerebral flow on computed tomography (CT) perfusion [56], or single-photon emission computed tomography (SPECT) imaging [57], and increased glucose uptake on fluorodeoxyglucose (FDG)-positron emission tomography (PET) [58, 59]. These changes often reversed with the resolution of IIC patterns $[55,58]$.

3) IIC patterns may be associated with metabolic biomarkers of neuronal injury. Periodic discharges as well as nonconvulsive seizures were temporally associated with metabolic crisis such as increased lactate/pyruvate ratio (LPR) and decreased glucose levels during cerebral microdialysis in patients with traumatic brain injury [60]. PDs $>2 \mathrm{~Hz}$ in frequency were associated a decrease in the partial pressure of oxygen in interstitial brain tissue, and were more likely to be associated with secondary neuronal injury from lower regional oxygen saturation [61].

4) IIC patterns on scalp EEG can be associated with intracranial seizures on intracranial recordings using depth EEG. Intracranial EEG with simultaneous scalp EEG recording demonstrated that up to $19 \%$ of intracranial seizures detected on depth EEG were associated with IIC patterns on scalp recordings [62]. 


\section{Risk of IIC patterns associated with epilepsy}

The risk of epilepsy associated with IIC patterns has been reported in several small case studies. Epilepsy was developed in $48.5 \%$ of patients with LPDs and electrographic seizures during a mean follow-up duration of 11.9 months [63]. Patients with LPDs had a hazard ratio of $7.7(95 \% \mathrm{CI}=2.9-20.7)$ of developing epilepsy in comparison of patients without periodic patterns [64, 65] LPDs superimposed with rhythmic activity and electrographic seizures have a higher risk of developing epilepsy [27, 65]. Among the different acute brain injury subtypes, IIC patterns with acute TBI were highly associated with posttraumatic epilepsy [66]. In patients with ischemic stroke, LPDs and sporadic epileptiform discharges are associated with higher risk of strokerelated epilepsy [67-70]. Overall 10 to $60 \%$ patients with LPDs go on to develop chronic epilepsy after hospital discharge $[15,19,65]$.

\section{Treatment algorithms}

There are no evidence-based guidelines on how to treat patients with IIC patterns, particularly on how aggressively to treat. A number of treatment algorithms have been proposed in the literature, which can be summarized in several different approaches $[9,51,53,71-74]$. Treatment decision should be guided by the previously discussed risk stratification on a case by case basis.

\section{Treat non-convulsive seizures}

The first step is to differentiate NCS from IIC patterns. Periodic discharges and rhythmic patterns occurring at frequency of $>2.5 \mathrm{~Hz}$, associated with spatiotemporal evolution and subtle ictal clinical accompaniments are classified as seizures and should be treated accordingly [4].

\section{Mitigate the seizure risk associated with IIC patterns} Most of IIC patterns (other than GRDA) are highly associated with increased risk of seizures, particularly when they are $>2.0 \mathrm{~Hz}$ and associated with "plus" features. It is critical that patients with IIC patterns should be monitored with continuous EEG for the surveillance of NCS and NCSE. Prophylactic treatment with non-sedating anti-seizure medications (ASM) such as Levetiracetam and Lacosamide may be considered [51]. The therapeutic goal of prophylaxis is to prevent IIC patterns from evolving into seizures as seen in Fig. 5, and is not intend to suppress IIC patterns.

\section{Treat IIC patterns as potential ictal patterns}

Given the paucity of the available evidence, it is highly controversial whether IIC patterns should be treated as potentially ictal patterns. When the treatment decision is uncertain, a trial of benzodiazepine or a loading dose of
ASM may be empirically performed $[8,75]$. If there are both immediate electrographic and clinical improvements, the trial is considered positive and further ASM treatment is warranted for pattern suppression. If there is an immediate electrographic improvement without clinical improvement, the trial is considered equivocal and patients should be further followed for the possible delayed clinical response. Neuronal injury using neuroimaging and biochemical markers may be further assessed for the consideration of treatment. During clinical practice, a positive trial of benzodiazepine or ASM is often not observed, because many critically ill patients have underlying altered mental status and encephalopathy, which may confound the clinical improvement in these patients.

Once treatment is initiated, the next step is to decide how aggressively to treat and endpoints. To prevent the potential neuronal injury associated with IIC patterns, the endpoint is to achieve pattern suppression. Conventional ASM with less sedation and few drug-drug interactions should be quickly titrated [68]. Based on EEG response, a second ASM can be added if clinical or electrographic improvement is not seen. More aggressively, intravenous anesthetics such as midazolam and propofol can be further considered if conventional ASM are not effective [74]. Nevertheless, several studies suggested that intravenous anesthetic uses may increase the poor outcomes and mortality in the patients with non-convulsive status epilepticus [76-78], which should service as a caution for the use of anesthetics to treat patients with IIC patterns. Moreover, some IIC patterns such as LPDs in patients with acute stroke and GPDs in patients with anoxic brain injury are highly refractory to the treatment of ASM and anesthetics. Therefore, the benefits of pattern suppression must be individualized and carefully weighed against the risk of iatrogenic complications such as cardiorespiratory depression [79]. Continuous EEG monitoring is essential to guide effective treatment and limit unnecessarily iatrogenic complications of ASM and anesthetic treatments.

\section{Chronic treatment of IIC related epilepsy}

Once patients are discharged from intensive care unit, the question is whether to continue to treat the patients with ASM. Some authors suggested that for patients with IIC patterns and without seizures during ICU admission, it is not unreasonable to discontinue the prophylactic ASM treatment at the time of hospital discharge. For patients with IIC patterns and seizures during ICU admission, ASM may be continued for 6-12 months [71]. For IIC patients with preexisting epilepsy and late onset seizures, long-term ASM treatment are often necessary. 


\section{Conclusion}

Ictal-interictal continuum presents a challenging electrophysiological and clinical conundrum in the management of critically ill patients. IIC patterns are neither ictal nor interictal, and their clinical significance remains uncertain. There are no evidence-based guidelines on how to treat the patients with IIC patterns. Treatment should be based on the patient's overall clinical picture and the seizure risk associated with IIC patterns. Prophylactic treatment with non-sedating ASM may be considered to mitigate the seizure risk for IIC patterns. When the treatment decision is uncertain, benzodiazepine trial or a loading dose of ASM can be empirically performed. If there are both electrographic and clinical improvements, further ASM treatment is warranted. Anesthetic agents may be considered when conventional ASM are not effective. If there is an immediate electrographic improvement without clinical improvement, patients should be further followed for the possible delayed clinical response. Neuronal injury using neuroimaging and biochemical markers may be further assessed for the consideration of treatment. Treatment decision should weigh the potential neuronal injury of IIC patterns against the iatrogenic complications of ASM. Regardless, treatment of underlying etiology is paramount in critically ill patients with IIC patterns.

\section{Abbreviations \\ ACNS: American Clinical Neurophysiology Society; ASM: Anti-seizure medications; BIPDs: Bilateral independent periodic discharges; BI- PLEDs: Bilateral independent periodic lateralized epileptiform discharges; B(I)RDs: Brief potentially ictal rhythmic discharges; cEEG: continuous electroencephalogram; CT: Computed tomography ; DWl: Diffusion weighted imaging; FDG: Fluorodeoxyglucose; FIRDA: Frontal intermittent rhythmic delta activity; GPEDs: Generalized periodic epileptiform discharges; GPDs: Generalized periodic discharges; GRDA: Generalized rhythmic delta activity; IC: Ictal-interictal continuum; LPDs: Lateralized periodic discharges; LPR: Lactate/pyruvate ratio; LRDA: Lateralized rhythmic delta activity; NCS: Non-convulsive seizures; NCSE: Non-convulsive status epilepticus; OIRDA: Occipital intermittent rhythmic delta activity; PD: Periodic discharges; PET: Positron emission tomography; PLEDs: Periodic lateralized epileptiform discharges; RDA: Rhythmic delta activity; SIRPIDs: Stimulus-induced rhythmic, periodic or ictal discharges; SPECT: Single-photon emission computed tomography}

\section{Acknowledgements}

N/A.

\section{Authors' contributions}

JXT: Conceived the study, reviewed literature and wrote the manuscript. XXO: Reviewed literature and the manuscript for intellectual context; QW: Reviewed literature and the manuscript for intellectual context. The author(s) read and approved the final manuscript.

\section{Authors' information}

James Tao, MD, PhD. Associate Professor in the Department of Neurology, the University of Chicago, IL. USA.

XiaoXiao Qin, MD. Epileptologist in the Department of Neurology, Beijing Tiantan Hospital, Capital Medical University, Beijing, P. R. China, and China National Clinical Research Center for Neurological Diseases, Beijing, P. R. China.
Qun Wang, MD, PhD. Professor of Department of Neurology, Beijing Tiantan Hospital, Capital Medical University, Beijing, P. R. China, and China National Clinical Research Center for Neurological Diseases, Beijing, P. R. China.

\section{Funding}

This work was supported by the Capital Healthy Development Research Funding 2020-1-2013 and 2016-1-2011.

Availability of data and materials

N/A. There was no original data in this study.

Ethics approval and consent to participate

$\mathrm{N} / \mathrm{A}$, this is systematic review of literature.

\section{Consent for publication}

All the authors are consent for the publication of this article.

\section{Competing interests}

Authors have no competing interests.

\section{Author details}

'Department of Neurology, The University of Chicago, 5841 South Maryland Ave. MC2030, Chicago, IL 6063, U.S.A.. ²Department of Neurology, Beijing Tiantan Hospital, Capital Medical University, Beijing, P. R. China. ${ }^{3}$ China National Clinical Research Center for Neurological Diseases, Beijing, P. R. China.

Received: 8 May 2020 Accepted: 1 July 2020

Published online: 31 August 2020

\section{References}

1. Cobb W, Hill D. Electroencephalogram in subacute progressive encephalitis. Brain. 1950;73:392-404

2. Chatrian GE, Shaw CM, Leffman $\mathrm{H}$. The significance of periodic lateralized Epileptiform discharges in Eeg: an electrographic, clinical and pathological study. Electroencephalogr Clin Neurophysiol. 1964;17:177-93.

3. Hirsch $\sqcup$, Brenner RP, Drislane FW, et al. The ACNS subcommittee on research terminology for continuous EEG monitoring: proposed standardized terminology for rhythmic and periodic EEG patterns encountered in critically ill patients. J Clin Neurophysiol. 2005;22:128-35.

4. Hirsch LJ, LaRoche SM, Gaspard N, et al. American clinical neurophysiology Society's standardized critical care EEG terminology: 2012 version. J Clin Neurophysiol. 2013;30:1-27.

5. Maciel CB, Hirsch LJ. Definition and classification of periodic and rhythmic patterns. J Clin Neurophysiol. 2018:35:179-88.

6. Pohlmann-Eden B, Hoch DB, Cochius II, et al. Periodic lateralized epileptiform discharges--a critical review. J Clin Neurophysiol. 1996;13: 519-30.

7. Chong DJ, Hirsch LJ. Which EEG patterns warrant treatment in the critically ill? Reviewing the evidence for treatment of periodic epileptiform discharges and related patterns. J Clin Neurophysiol. 2005;22:79-91.

8. Sivaraju A, Gilmore EJ. Understanding and managing the Ictal-Interictal continuum in Neurocritical care. Curr Treat Options Neurol. 2016;18:8.

9. Cormier J, Maciel CB, Gilmore EJ. Ictal-Interictal continuum: when to worry about the continuous electroencephalography pattern. Semin Respir Crit Care Med. 2017:38:793-806.

10. Yoo JY, Rampal N, Petroff OA, et al. Brief potentially ictal rhythmic discharges in critically ill adults. JAMA Neurol. 2014;71:454-62.

11. Claassen J, Mayer SA, Kowalski RG, et al. Detection of electrographic seizures with continuous EEG monitoring in critically ill patients. Neurology. 2004;62: 1743-8.

12. Fitzpatrick W, Lowry N. PLEDs: clinical correlates. Can J Neurol Sci. 2007;34: 443-50

13. Orta DS, Chiappa KH, Quiroz AZ, et al. Prognostic implications of periodic epileptiform discharges. Arch Neurol. 2009;66:985-91.

14. Gaspard N, Hirsch LJ. Pitfalls in ictal EEG interpretation: critical care and intracranial recordings. Neurology. 2013:80:S26-42.

15. Garcia-Morales I, Garcia MT, Galan-Davila L, et al. Periodic lateralized epileptiform discharges: etiology, clinical aspects, seizures, and evolution in 130 patients. J Clin Neurophysiol. 2002;19:172-7. 
16. Newey CR, Sahota P, Hantus S. Electrographic features of lateralized periodic discharges stratify risk in the interictal-Ictal continuum. J Clin Neurophysiol. 2017;34:365-9.

17. Li HT, Wu T, Lin WR, et al. Clinical correlation and prognostic implication of periodic EEG patterns: a cohort study. Epilepsy Res. 2017;131:44-50.

18. Claassen J, Jette N, Chum F, et al. Electrographic seizures and periodic discharges after intracerebral hemorrhage. Neurology. 2007;69:1356-65.

19. Walsh JM, Brenner RP. Periodic lateralized epileptiform discharges--longterm outcome in adults. Epilepsia. 1987;28:533-6.

20. Hartings JA, Williams AJ, Tortella FC. Occurrence of nonconvulsive seizures, periodic epileptiform discharges, and intermittent rhythmic delta activity in rat focal ischemia. Exp Neurol. 2003;179:139-49.

21. Gaspard N, Manganas L, Rampal N, et al. Similarity of lateralized rhythmic delta activity to periodic lateralized epileptiform discharges in critically ill patients. JAMA Neurol. 2013;70:1288-95.

22. Rodriguez Ruiz A, Vlachy J, Lee JW, et al. Association of periodic and rhythmic electroencephalographic patterns with Seizures in critically ill patients. JAMA Neurol. 2017;74:181-8.

23. Kurtz P, Gaspard N, Wahl AS, et al. Continuous electroencephalography in a surgical intensive care unit. Intensive Care Med. 2014;40:228-34.

24. Sainju RK, Manganas LN, Gilmore EJ, et al. Clinical correlates and prognostic significance of lateralized periodic discharges in patients without acute or progressive brain injury: a case-control study. J Clin Neurophysiol. 2015;32: 495-500.

25. Foreman B, Claassen J, Abou Khaled K, et al. Generalized periodic discharges in the critically ill: a case-control study of 200 patients. Neurology. 2012;79: 1951-60.

26. Sully KE, Husain AM. Generalized periodic discharges: a topical review. J Clin Neurophysiol. 2018;35:199-207.

27. Pedersen GL, Rasmussen SB, Gyllenborg J, et al. Prognostic value of periodic electroencephalographic discharges for neurological patients with profound disturbances of consciousness. Clin Neurophysiol. 2013; 124:44-51.

28. Husain AM, Mebust KA, Radtke RA. Generalized periodic epileptiform discharges: etiologies, relationship to status epilepticus, and prognosis. J Clin Neurophysiol. 1999;16:51-8.

29. Struck AF, Osman G, Rampal N, et al. Time-dependent risk of seizures in critically ill patients on continuous electroencephalogram. Ann Neurol. 2017; 82:177-85.

30. Raroque HG Jr, Purdy P. Lesion localization in periodic lateralized epileptiform discharges: gray or white matter. Epilepsia. 1995;36:58-62.

31. de la Paz D, Brenner RP. Bilateral independent periodic lateralized epileptiform discharges. Clinical significance. Arch Neurol. 1981;38:713-5.

32. Schmitt SE. Generalized and lateralized rhythmic patterns. J Clin Neurophysiol. 2018;35:218-28.

33. Gambardella A, Gotman J, Cendes F, et al. Focal intermittent delta activity in patients with mesiotemporal atrophy: a reliable marker of the epileptogenic focus. Epilepsia. 1995;36:122-9.

34. Tao JX, Chen XJ, Baldwin M, et al. Interictal regional delta slowing is an EEG marker of epileptic network in temporal lobe epilepsy. Epilepsia. 2011;52: 467-76

35. Di Gennaro G, Quarato PP, Onorati P, et al. Localizing significance of temporal intermittent rhythmic delta activity (TIRDA) in drug-resistant focal epilepsy. Clin Neurophysiol. 2003;114:70-8.

36. Kubota F, Ohnishi N. Study on FIRDA and $3 \mathrm{~Hz}$ rhythmic slow wave bursts occurring in the frontal area of epileptic patients. Clin Electroencephalogr. 1997;28:112-6.

37. Swisher CB, Shah D, Sinha SR, et al. Baseline EEG pattern on continuous ICU EEG monitoring and incidence of seizures. J Clin Neurophysiol. 2015;32:147-51.

38. Dericioglu N, Khasiyev F, Arsava EM, et al. Frontal Intermittent Rhythmic Delta Activity (FIRDA) in the neurological intensive care: prevalence, determinants, and clinical significance. Clin EEG Neurosci. 2018;49:272-7.

39. Sutter R, Stevens RD, Kaplan PW. Clinical and imaging correlates of EEG patterns in hospitalized patients with encephalopathy. J Neurol. 2013;260: 1087-98.

40. Crepeau AZ, Kerrigan JF, Gerber P, et al. Rhythmical and periodic EEG patterns do not predict short-term outcome in critically ill patients with subarachnoid hemorrhage. J Clin Neurophysiol. 2013;30:247-54.

41. Hirsch L, Claassen J, Mayer SA, et al. Stimulus-induced rhythmic, periodic, or ictal discharges (SIRPIDs): a common EEG phenomenon in the critically ill. Epilepsia. 2004;45:109-23.
42. Braksick SA, Burkholder DB, Tsetsou S, et al. Associated factors and prognostic implications of stimulus-induced rhythmic, periodic, or ictal discharges. JAMA Neurol. 2016;73:585-90.

43. Ong C, Gilmore E, Claassen J, et al. Impact of prolonged periodic epileptiform discharges on coma prognosis. Neurocrit Care. 2012;17:39-44.

44. Johnson EL, Kaplan PW, Ritzl EK. Stimulus-induced rhythmic, periodic, or ictal discharges (SIRPIDs). J Clin Neurophysiol. 2018;35:229-33.

45. Shewmon DA. What is a neonatal seizure? Problems in definition and quantification for investigative and clinical purposes. J Clin Neurophysiol. 1990;7:315-68.

46. Oliveira AJ, Nunes ML, Haertel LM, et al. Duration of rhythmic EEG patterns in neonates: new evidence for clinical and prognostic significance of brief rhythmic discharges. Clin Neurophysiol. 2000;111:1646-53.

47. Nagarajan L, Palumbo L, Ghosh S. Brief electroencephalography rhythmic discharges (BERDs) in the neonate with seizures: their significance and prognostic implications. J Child Neurol. 2011;26:1529-33.

48. Passareli V, Medeiros L, Simabukuro M, et al. Early occurrence of brief potentially ictal rhythmic discharges [B(I)RDs], and subsequent emergence of asymmetric extreme delta brush (EDB) in anti-NMDA receptors encephalitis. Arq Neuropsiquiatr. 2016;74:177.

49. Yoo JY, Marcuse LV, Fields MC, et al. Brief potentially ictal rhythmic discharges [B(I)RDs] in noncritically ill adults. J Clin Neurophysiol. 2017;34:222-9.

50. Leitinger M, Beniczky S, Rohracher A, et al. Salzburg consensus criteria for non-convulsive status epilepticus--approach to clinical application. Epilepsy Behav. 2015;49:158-63.

51. Osman GM, Araujo DF, Maciel CB. Ictal Interictal continuum patterns. Curr Treat Options Neurol. 2018;20:15

52. Yemisci M, Gurer G, Saygi S, et al. Generalised periodic epileptiform discharges: clinical features, neuroradiological evaluation and prognosis in 37 adult patients. Seizure. 2003;12:465-72.

53. Zafar SF, Subramaniam T, Osman G, et al. Electrographic seizures and ictalinterictal continuum (IIC) patterns in critically ill patients. Epilepsy Behav. 2020;106:107037.

54. Sen-Gupta I, Schuele SU, Macken MP, et al. "Ictal" lateralized periodic discharges. Epilepsy Behav. 2014;36:165-70.

55. Claassen J. How I treat patients with EEG patterns on the ictal-interictal continuum in the neuro ICU. Neurocrit Care. 2009;11:437-44.

56. Royter V, Paletz L, Waters MF. Stroke vs. status epilepticus. A case report utilizing CT perfusion. J Neurol Sci. 2008;266:174-6.

57. Assal F, Papazyan JP, Slosman DO, et al. SPECT in periodic lateralized epileptiform discharges (PLEDs): a form of partial status epilepticus? Seizure. 2001;10:260-5.

58. Handforth A, Cheng JT, Mandelkern MA, et al. Markedly increased mesiotemporal lobe metabolism in a case with PLEDs: further evidence that PLEDs are a manifestation of partial status epilepticus. Epilepsia. 1994;35: 876-81.

59. Struck AF, Westover MB, Hall LT, et al. Metabolic correlates of the ictalinterictal continuum: FDG-PET during continuous EEG. Neurocrit Care. 2016; 24:324-31.

60. Vespa P, Tubi M, Claassen J, et al. Metabolic crisis occurs with seizures and periodic discharges after brain trauma. Ann Neurol. 2016;79:579-90.

61. Witsch J, Frey HP, Schmidt JM, et al. Electroencephalographic periodic discharges and frequency-dependent brain tissue hypoxia in acute brain injury. JAMA Neurol. 2017;74:301-9.

62. De Marchis GM, Lantigua H, Schmidt JM, et al. Impact of premorbid hypertension on haemorrhage severity and aneurysm rebleeding risk after subarachnoid haemorrhage. J Neurol Neurosurg Psychiatry. 2014;85:56-9.

63. Punia V, Vakani $R$, Burgess $R$, et al. Electrographic and clinical natural history of lateralized periodic discharges. J Clin Neurophysiol. 2018;35:71-6.

64. Newey CR, Kinzy TG, Punia V, et al. Continuous electroencephalography in the critically ill: clinical and continuous electroencephalography markers for targeted monitoring. J Clin Neurophysiol. 2018;35:325-31.

65. Punia $V$, Garcia CG, Hantus S. Incidence of recurrent seizures following hospital discharge in patients with LPDs (PLEDs) and nonconvulsive seizures recorded on continuous EEG in the critical care setting. Epilepsy Behav. 2015:49:250-4.

66. Kim JA, Boyle EJ, Wu AC, et al. Epileptiform activity in traumatic brain injury predicts post-traumatic epilepsy. Ann Neurol. 2018;83:858-62.

67. De Reuck J, Goethals M, Claeys I, et al. EEG findings after a cerebral territorial infarct in patients who develop early- and late-onset seizures. Eur Neurol. 2006;55:209-13. 
68. De Reuck J, Hemelsoet D, Van Maele G. Seizures and epilepsy in patients with a spontaneous intracerebral haematoma. Clin Neurol Neurosurg. 2007; 109:501-4.

69. Bentes $\mathrm{C}$, Martins H, Peralta AR, et al. Early EEG predicts poststroke epilepsy. Epilepsia Open. 2018;3:203-12.

70. Bentes C, Martins H, Peralta AR, et al. Post-stroke seizures are clinically underestimated. J Neurol. 2017;264:1978-85.

71. Jirsch J, Hirsch $\amalg$. Nonconvulsive seizures: developing a rational approach to the diagnosis and management in the critically ill population. Clin Neurophysiol. 2007;118:1660-70.

72. Petrone P, Rodriguez Velandia W, Dziakova J, et al. Treatment of complex perineal trauma. A review of the literature. Cir Esp. 2016;94:313-22.

73. Kalamangalam GP, Pohlmann-Eden B. Ictal-Interictal Continuum. J Clin Neurophysiol. 2018;35:274-8.

74. Rubinos C, Reynolds AS, Claassen J. The ictal-interictal continuum: to treat or not to treat (and how)? Neurocrit Care. 2018;29:3-8.

75. Herlopian A, Struck AF, Rosenthal E, et al. Neuroimaging correlates of periodic discharges. J Clin Neurophysiol. 2018;35:279-94.

76. Litt B, Wityk RJ, Hertz SH, et al. Nonconvulsive status epilepticus in the critically ill elderly. Epilepsia. 1998;39:1194-202.

77. Marchi NA, Novy J, Faouzi M, et al. Status epilepticus: impact of therapeutic coma on outcome. Crit Care Med. 2015:43:1003-9.

78. Sutter R, Marsch S, Fuhr P, et al. Anesthetic drugs in status epilepticus: risk or rescue? A 6-year cohort study. Neurology. 2014:82:656-64.

79. Rodriguez V, Rodden MF, LaRoche SM. Ictal-interictal continuum: a proposed treatment algorithm. Clin Neurophysiol. 2016;127:2056-64

Ready to submit your research? Choose BMC and benefit from:

- fast, convenient online submission

- thorough peer review by experienced researchers in your field

- rapid publication on acceptance

- support for research data, including large and complex data types

- gold Open Access which fosters wider collaboration and increased citations

- maximum visibility for your research: over $100 \mathrm{M}$ website views per year

At $\mathrm{BMC}$, research is always in progress.

Learn more biomedcentral.com/submissions 